\title{
Underlying mechanisms of the self-sustained oscillation of a nanomechanical stochastic resonator in a liquid
}

\author{
J. Tamayo,* M. Calleja, D. Ramos, and J. Mertens \\ Instituto de Microelectrónica de Madrid, IMM-CNM (CSIC), Isaac Newton 8 (PTM), Tres Cantos, 28760 Madrid, Spain
}

(Received 30 May 2007; revised manuscript received 3 September 2007; published 1 November 2007)

\begin{abstract}
We study the stochastic response of a nanomechanical resonator in a liquid connected to a feedback loop to increase the quality $(Q)$ factor. We distinguish two regimes in the oscillation. Below a critical value of the feedback gain, an effective increase of the $Q$ factor and temperature is produced. When the gain surpasses the critical value, the resonator initially behaves as having negative damping until the saturation of the system comes into play and a steady situation is reached. In this regime, frequency stabilities of about 1 part per $10^{6}$ can be achieved.
\end{abstract}

DOI: 10.1103/PhysRevB.76.180201

PACS number(s): 62.10.+s, 46.40.-f, 05.40.-a, 46.70.De

The intimate connection between the oscillation of nanomechanical resonators and the near environment has set the basis for a number of relevant applications that include scanning probe microscopies and sensors. Temperature, ${ }^{1}$ fluid rheological properties, ${ }^{2}$ molecular forces, ${ }^{3}$ single-particle spin, ${ }^{4}$ and molecular adsorption ${ }^{5,6}$ are stimuli that can largely influence the resonant properties of the oscillator and then be measured. The operation of nanomechanical resonators in aqueous environments has allowed the exploration and detection of biological systems in their native environment. Thus, in atomic force microscopy (AFM), a vibrating microcantilever with a built-in sharp tip immersed in aqueous solution is used to gently probe the surface properties and topography of biological specimens with nanometer-scale spatial resolution. ${ }^{7,8}$ The resonant frequency of the cantilever shifts as a consequence of the tip-sample interactions. In other applications, microcantilever resonators sensitized with molecular receptors are used for in situ specific detection of biological samples such as DNA, proteins, and pathogens. ${ }^{9}$ The concept here is that the resonant frequency sensitively displaces when molecules land on the cantilever surface. Depending on the sensor design, the resonant frequency change can be due to the added mass, the adsorbate stiffness, or both. ${ }^{10,11}$

Further progress of these applications is being hampered by the large mechanical energy loss produced when the resonator vibrates in a liquid. ${ }^{12}$ This causes an extremely low $Q$ factor that largely limits the sensitivity of the resonant frequency to external stimuli. This limitation has motivated the design of resonators with new geometries ${ }^{13,14}$ as well as the use of positive feedback loops. ${ }^{12,15-18}$ The aim of any feedback loop is to modify a dynamical system to create a "better" one. They are used in diverse areas such as chaos, statistical mechanics, optics, quantum mechanics, and genetic networks. ${ }^{19,20}$ The basic principle of the feedback loops used to enhance the sensitivity of resonators in liquids is driving the resonator with a signal roughly proportional to its velocity in order to counteract the viscous damping. ${ }^{12}$ The application of this technique has provided unprecedented molecular resolution in AFM in liquids ${ }^{16,17}$ and promising results for in situ biological detection by using nanomechanical resonators. ${ }^{21}$ Moreover, this technique has recently been applied for self-excitation of the motion of a single electron, which opens the way to improved measurements of the mag- netic moment of single particles. ${ }^{22}$ Despite these important applications, it has not been established how the physical properties of the resonator and feedback parameters influence the frequency resolution of the system. ${ }^{23,24}$

The present work provides a physical model validated by numerical simulations to understand the dynamics of selfsustained stochastic nanomechanical resonators in liquids.

Basically, in a feedback loop to enhance the $Q$ factor, the output of the sensor that measures the resonator oscillation is amplified $G$ times by a variable gain amplifier, shifted $\phi$ radians by a variable phase shifter, and then connected to the input of the actuator that provides an excitation force to the resonator. The closed loop acts in the sources of noise of the system-namely, the thermomechanical fluctuations mediated by collisions between the molecules in the medium and the resonator and the electrical noise from the displacement sensor. For the sake of a major understanding, we assume that the thermomechanical fluctuations dominate the system noise. ${ }^{25}$ The differential equation of the motion is given by

$$
\left[m\left(d^{2} / d t^{2}\right)+\gamma(d / d t)+k\right] z(t)=F_{\mathrm{th}}(t)+F_{\mathrm{fb}}(z),
$$

where $m$ is the effective mass of the resonator, $\gamma$ is the damping constant, and $k$ is the spring constant. The resonant frequency is given by $\omega_{0}=(\mathrm{k} / \mathrm{m})^{1 / 2}$ and the $Q$ factor by $Q$ $=m \omega_{0} / \gamma . F_{\text {th }}$ represents the random Langevin force whose autocorrelation function spectral density is approximately flat and given by $\Psi_{\text {th }}=4 \gamma k_{B} T$ where $k_{B}$ is the Boltzmann constant and $T$ is the absolute temperature. ${ }^{26,27}$ Notice that effective mass and the damping constant are frequency dependent in liquids due to the hydrodynamic force. For the sake of major understanding, we have assumed constant values as the self-excitation phenomenology occurs in a narrow frequency range around the resonance. ${ }^{28} F_{\mathrm{fb}}(z)$ represents the feedback force that is $G e^{i \phi} z(t)$ except when the feedback force exceeds the maximum force tolerated by the actuator, $F_{s}$. For self-sustained oscillations in which $\left|F_{\mathrm{fb}}(z)\right|<F_{s}$, the resonator response is characterized by its mechanical susceptibility, ${ }^{25}$

$\left.X_{\mathrm{eff}}(\omega)=\left\{\left[(k-G \cos \phi)-m \omega^{2}\right]+i[\gamma \omega-G \sin \phi)\right]\right\}^{-1}$.

By adjusting $\phi=\pi / 2$, the feedback loop reduces the effective damping of the system to $\gamma_{\mathrm{eff}} \cong \gamma-G / \omega_{0}$ without changing the resonant frequency. The linewidth of the 


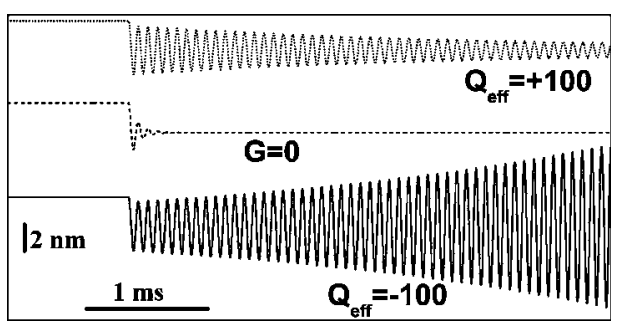

(a)

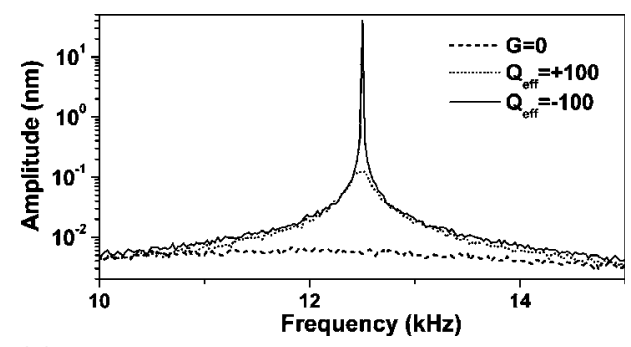

(b)

FIG. 1. Simulation data of the stochastic properties of a nanomechanical resonator by measuring (a) the transient oscillation when an applied force of $5 \mathrm{nN}$ is removed and (b) the noise frequency spectra of the system in the steady state. The parameters used are $k=0.4 \mathrm{~N} / \mathrm{m}, \omega_{0} /(2 \pi)=12500 \mathrm{~Hz}$, and $Q=3$. The data are calculated for the feedback loop gain open (dotted lines), $Q_{\text {eff }}$ $=100$ (dashed lines), and $Q_{\text {eff }}=-100$ (solid lines).

resonator frequency response is consequently reduced to $\sim \omega_{0} / Q_{\text {eff }}$, where the effective $Q$ factor is $Q_{\text {eff }} \cong m \omega_{0} / \gamma_{\text {eff. }}$.

Figure 1 illustrates how the dynamics of a microcantilever in liquid with $Q=3, k=0.4 \mathrm{~N} / \mathrm{m}$, and $\omega_{0}=2 \pi \times 12.5 \mathrm{kHz}$ at $T=300 \mathrm{~K}$ is changed by the feedback loop. The simulations have been performed by numerically solving Eq. (1) by a fourth-order Runger-Kutta method. The Langevin force was supplied by a Gaussian random number generator, in consistency with the fluctuation-dissipation theorem. The scheme used to measure the stochastic properties of the resonator is as follows: (i) apply a constant force $(5 \mathrm{nN})$ during a sufficiently long time, then turn it off and measure how the stored mechanical energy is released to the medium until the system reaches its steady $\operatorname{state}^{27}$ [Fig. 1(a)], and (ii) measure the frequency distribution of the noise of the system in the steady state [Fig. 1(b)]. When the feedback loop is open, the cantilever releases most of the stored mechanical energy in a couple of cycles [Fig. 1(a), dashed line]. Due to the low $Q$ factor, the amplitude spectrum of the noise is quite flat around the resonance frequency [Fig. 1(b), dashed line]. If the feedback loop is closed and the feedback gain is adjusted to obtain an effective $Q$ factor of 100, the transient oscillation exponentially decreases with a decay time of $\cong 2 Q_{\text {eff }} / \omega_{0}$ [Fig. 1(a), dotted line]. Correspondingly, the noise linewidth is considerably reduced [Fig. 1(b), dotted line]. More intriguingly, if the gain is still increased in order to reverse the sign of the effective $Q$ factor, the transient oscillation exponentially increases (rise time $\cong 2 Q_{\text {eff }} / \omega_{0}$ ) [Fig. 1(a), solid line]. The amplitude grows until the nonlinear saturation response of the actuator comes into play and the system achieves a steady state whose amplitude spectrum exhibits a very nar-

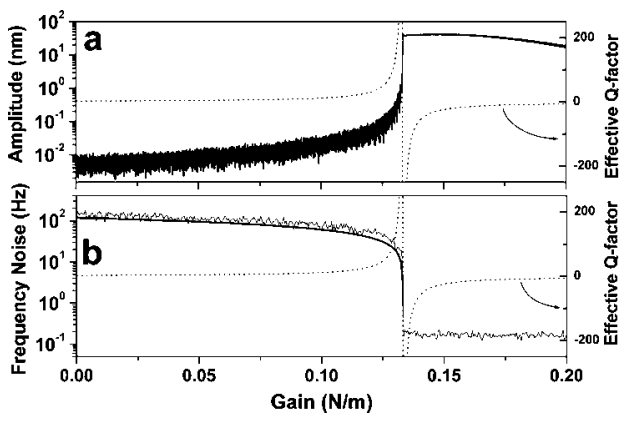

FIG. 2. Simulation data of the mean and standard deviation of the oscillation amplitude (a) and frequency noise (b) as a function of the feedback loop gain. The bandwidth is $\Delta \omega /(2 \pi) \cong 12 \mathrm{~Hz}$, and the resonator parameters are the same as those in Fig. 1. The statistics are obtained from 32 independent simulations. The effective $Q$ factor is also plotted in both graphs (dotted lines). Thick line in (b) represents the analytical theory developed in the text.

row linewidth around the resonance frequency [Fig. 1(b), solid line].

In Fig. 2(a), we plot the numerical calculations of the mean and standard deviation values of the amplitude at resonance as a function of the feedback loop gain. For a major understanding, the effective $Q$ factor is also plotted. The amplitude statistics is obtained from 32 independent ensembles for a measurement bandwidth of $12 \mathrm{~Hz}$. We distinguish two regimes in the oscillation behaviour that are related to the sign of the effective $Q$ factor. For gains below the critical value $G_{c}=Q / k$, the effective $Q$ factor is positive. The amplitude is proportional to the effective $Q$ factor, and it only grows appreciably for gains near the critical gain. The signal to noise remains constant with a small value of 1.9 , approximately. For $G \geqslant G_{c}$ the effective $Q$ factor is negative and the amplitude undergoes an abrupt increase of about two orders of magnitude. The amplitude is approximately given by $A_{s}$ $=Q F_{s} / k$. When the gain exceeds about $30 \%$ of $G_{c}$, the amplitude starts to decline due to the generation of higher harmonics. The signal-to-noise ratio in this regime achieves 900-1000. The frequency noise versus the gain is shown in Fig. 2(b). The frequency noise is moderately enhanced with the gain in the regime of positive $Q$ factors, in particular for values near $G_{c}$. Thus, the frequency noise is of about $150 \mathrm{~Hz}$ for the open feedback loop, whereas is of about $30 \mathrm{~Hz}$ for an effective $Q$-factor of 100 . However, when $G \geqslant G_{c}$, the frequency noise sharply decreases to $\approx 0.15 \mathrm{~Hz}$. In addition, the frequency noise shows negligible dependence on the gain.

The oscillation with $Q_{\text {eff }}>0$ can be characterized by its mechanical susceptibility; hence, the spectral density of the noise autocorrelation function is given by $S_{z}(\omega)$ $=\left|X_{\text {eff }}(\omega)\right|^{2} \psi_{\text {th }}$. Since the motion of the molecules that surround the resonator is not modified by the feedback loop, $\psi_{\text {th }}$ is not changed. Satisfaction of the fluctuation-dissipation theorem then produces the effect that the resonator is at a higher effective temperature given by $T_{\text {eff }} / T=Q_{\text {eff }} / Q .{ }^{20}$ Let us now consider an interval $\Delta t$, in which the noise $z(t)$ is assumed to have zero average value. The noise can then be expanded in Fourier series and be conveniently written as $z(t)=r(t) \cos \left[\omega_{0} t+\theta(t)\right], \quad$ where $\quad r^{2}(t)=x^{2}(t)+y^{2}(t) \quad$ and $\tan \theta(t)=y(t) / x(t)$ and 

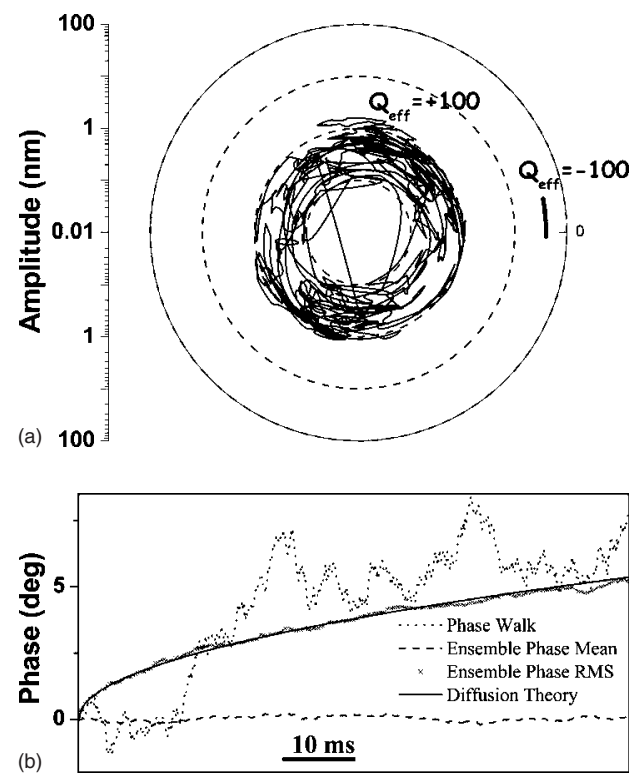

FIG. 3. (a) Polar graph of the self-sustained oscillations for values of the effective $Q$ factor of 100 and -100 . (b) Random phase walk (dotted line), ensemble average of the phase (dashed line), ensemble root mean square (crosses), and diffusion theory (solid line; see related text). The ensemble statistics is obtained from 128 independent simulations and $\Delta \omega /(2 \pi) \cong 12 \mathrm{~Hz}$. The resonator parameters are those used in Fig. 1.

$$
\begin{aligned}
& x(t)=\sum_{k} \sqrt{\frac{1}{\pi} S_{z}\left(\omega_{k}\right) \Delta \omega} \cos \left[\left(\omega_{k}-\omega_{0}\right) t+\alpha_{k}\right], \\
& y(t)=-\sum_{k} \sqrt{\frac{1}{\pi} S_{z}\left(\omega_{k}\right) \Delta \omega} \sin \left[\left(\omega_{k}-\omega_{0}\right) t+\alpha_{k}\right],
\end{aligned}
$$

where $k=1,2,3, \ldots, \omega_{k}=2 \pi k / \Delta t$, and $\alpha_{k}$ are uniformly distributed random phase angles that ensure that $z(t)$ is Gaussian distributed. It is easy to check that the autocorrelation function spectral density of $z(t)$ is given by $S_{z}$ by taking the limit $\Delta t \rightarrow \infty$. The phase fluctuations can be viewed as frequency fluctuations by applying the relation $\delta \omega(t) \equiv \omega(t)-\omega_{0}$ $=d \theta / d t$, where $\omega(t)$ is the instantaneous frequency. For sufficiently long intervals $\Delta \omega=2 \pi / \Delta t \ll \omega_{0}$, the calculation of the ensemble average of the square frequency fluctuation gives

$$
\left\langle\delta \omega(t)^{2}\right\rangle=\frac{1}{2} \frac{\Delta \omega}{\omega_{0}} \frac{\int_{\omega_{0}-B}^{\omega_{0}+B} S_{z}(\omega)\left(\omega-\omega_{0}\right)^{2} d \omega}{\int_{\omega_{0}-B}^{\omega_{0}+B} S_{z}(\omega) d \omega} .
$$

Note that Eq. (4) distinguishes the measurement bandwidth $\Delta \omega$ from the bandpass filter width $B$ used in the measurement. This expression is plotted in Fig. 2(b) showing an excellent agreement with the simulation data. Equation (4) simply reduces to $\cong B \Delta \omega /\left(2 \pi Q_{\text {eff }}\right)$ for $\omega_{0} / Q_{\text {eff }} \ll \Delta \omega \ll \omega_{0}$.

The different dynamic behavior of resonators with positive and negative effective damping is illustrated in Fig. 3(a), which shows a polar representation of the noise amplitude $r(t)$ and phase $\theta(t)$ for $Q_{\text {eff }}=100$ and $Q_{\text {eff }}=-100$ during a time span of $80 \mu \mathrm{s}$. The oscillation with positive effective damping shows a large noise equally distributed in amplitude and phase, equivalent to the thermomechanical noise of a resonator with $Q=100$ and $T=10^{4} \mathrm{~K}$. However, the oscillation with negative damping has small fluctuations in the radial direction (amplitude) due to the strong nonlinear potential imposed by the saturation of the actuator and the tendency of the amplitude to grow due to the negative decay time of the transient. Moreover, the phase shows little variation from its initial value. The phase trajectory, plotted in Fig. 3(b) (dotted line), resembles a random walk process. From 128 statistically independent ensembles, we observe that the mean value (dashed line) is zero and the standard deviation (crosses) is approximately proportional to $t^{1 / 2}$, which is indicative of a diffusion process driven by white noise.

Based on the simulation results, the oscillation with negative damping can be modelled as one with zero amplitude noise and zero-mean stationary random phase-i.e., $z(t)$ $\cong A_{s} \exp \left\{i\left[\omega_{0} t+\theta(t)\right]\right\} \quad$ (recall $\left.A_{s} \cong Q F_{s} / k\right)$. The feedback force can be approximated as $i F_{s} \exp \left\{i\left[\omega_{0} t+\theta(t)\right]\right\}$, where $i$ $=(-1)^{1 / 2}$. By substituting in Eq. (1), one reaches, for $d \theta / d t$ $\ll \omega_{0}$,

$$
\begin{aligned}
m \frac{d \Omega(t)}{d t}+\left(\gamma+2 i m \omega_{0}\right) \Omega(t)= & -i \frac{\gamma \omega_{0}^{2}}{2 \pi F_{s}} \int_{t-\pi / \omega_{0}}^{t+\pi / \omega_{0}} F_{t h}(\tau) \\
& \times \exp \left\{-i\left[\omega_{0} \tau+\theta(\tau)\right]\right\} d \tau .
\end{aligned}
$$

Here, we have introduced the parameter $\Omega(t)=\left(\omega_{0} / 2 \pi\right)$ $\times\left[\theta\left(t+\pi / \omega_{0}\right)-\theta\left(t-\pi / \omega_{0}\right)\right]$, which represents the frequency deviation averaged over a oscillation period. Notice that $\omega_{0}$ $+\Omega(t)$ represents the inverse of the time interval between two consecutive zero crossings with the same velocity sign and hence represents a measurable magnitude of a stochastic oscillation. By writing the Langevin force as a function of its Fourier transform and by calculating the ensemble average of the modulus of both equation sides, we derive the frequency noise

$$
\left(\frac{\left\langle\Omega^{2}(t)\right\rangle}{\omega_{0}^{2}}\right)=g \frac{k_{B} T k}{Q^{3} F_{s}^{2}},
$$

where $g$ is given by

$$
g=\frac{2}{\pi^{3}} \int_{0}^{\infty} \frac{\sin ^{2}(\pi x)}{(x-1)^{2}\left(x^{2}+4+1 / Q^{2}\right)} d x .
$$

The parameter $g$ shows little dependence on the intrinsic quality factor, being of about 0.1139 for $Q=3$ and rapidly tending to 0.1164 as $Q$ increases. If a bandpass filter is used, the integral limits must be replaced by $1-B / \omega_{0}$ and 1 $+B / \omega_{0}$.

Equation (5) resembles the Langevin equation except that (i) the resistive coefficient is complex, $\gamma+2 i m \omega_{0}$, and (ii) the random force term on the right-hand side is correlated for times smaller than the fundamental period, $2 \pi / \omega_{0}$ (much 
smaller than the experimental time scales). Despite these differences, the solution of Eq. (5) for times $t \gg Q / \omega_{0}$ can be resolved in a similar manner to the Langevin equation; once that is known, the frequency noise [Eq. (6)]

$$
\left\langle\Theta^{2}(t)\right\rangle=2\left(D / A_{s}^{2}\right) t
$$

where the phase $\Theta(t)$ satisfies $d / d t \Theta(t)=\Omega(t)$ and the diffusion coefficient is given by

$$
D=g / Q\left(k_{B} T / \gamma\right) .
$$

Equation (8) confirms that the phase fluctuations are analogous to the diffusion of a Brownian particle that freely moves along a circular path of radius $A_{s}$. However, there exist important differences. Thus, the phase diffusion constant is $g / Q$ times the diffusion constant of a Brownian particle given by $D=k_{B} T / \gamma$. These differences can be reconciled by setting the effective temperature of the resonator to $T_{\text {eff }} / T=g / Q$. We plot in Fig. 3(b) the root ensemble-mean square of the phase noise given by Eqs. (8) and (9) (solid line), which shows excellent agreement with the simulation results (crosses). The coherence of the oscillations can be then improved by minimizing the diffusion coefficient and increasing the amplitude $A_{s}$.

We can now estimate the frequency stability that can be achieved in liquid environments. The frequency noise in the negative damping regime is given by $\left\langle\delta \omega(t)^{2}\right\rangle$ $=\left\langle\Omega^{2}(t)\right\rangle \Delta \omega / \omega_{0}$ where $\left\langle\Omega^{2}(t)\right\rangle$ is shown in Eq. (6). Examination of Eq. (6) identifies the critical resonator parameters to reduce the frequency noise. In order of importance, these are a high quality factor and low elastic stiffness. For the resonator parameters used in this work, a bandwidth of $1 \mathrm{~Hz}$ and a saturation force $F_{s}$ of about $20 \mathrm{nN}$, a frequency stability of about 1 part per $10^{6}$ can be achieved in ideal conditions; i.e., the only source of noise is the Langevin force. Now we can consider the implications of this frequency stability in the sensitivity of two of the most extended applications of nanomechanical resonators: resonant mass sensors and AFM. In the first application, the resonator has an active mass deduced from $m=k / \omega_{0}^{2}$ of $65 \mathrm{ng}$. The frequency stability would provide a mass sensitivity of about tens of femtograms in liquids that is of about four orders of magnitude better than the resolution obtained with conventional microcantilevers in liquid. ${ }^{9}$ Mass sensitivities of attograms could be obtained by simply reducing the resonator size. In AFM applications, force gradients between the tip and sample are translated into resonance frequency shifts. The use of selfsustained oscillations would allow sensitivities of the order of $100 \mathrm{aN}$ per nanometer that could, for instance, be applied for the study of protein unfolding with unprecedented resolution. ${ }^{29}$

In conclusion, we have developed a theory that unveils the underlying mechanisms of the oscillation of selfsustained nanomechanical resonators with low $Q$ factor. We distinguish two regimes: for feedback loop gains smaller than $G_{c}=Q / k$, the effect of the feedback on the stochastic resonator response is the effective increase of the $Q$ factor and the system temperature by the same factor. When the gain surpasses $G_{c}$, the oscillation enters upon a different dynamic behavior. Initially, the nanomechanical resonator behaves as having negative damping, and hence the transient oscillation exponentially grows until the actuator saturation comes into play and a steady situation is reached. At that point the oscillation can be described by the Brownian diffusion theory for a particle with the mass and damping constant of the resonator that can freely move along a circular path with radius given by the oscillation amplitude and with temperature proportional to the room temperature divided by the intrinsic $Q$ factor. An important consequence of our study is that self-sustained stochastic nanomechanical resonators in liquid can achieve frequency stabilities of about 1 part per $10^{6}$ which open a wide range of fascinating applications of nanomechanical resonators for exploration and detection of biological systems.

This research was supported by the Spanish Ministry of Science under Grant No. TEC2006-10316.

\footnotetext{
*jtamayo@imm.cnm.csic.es

${ }^{1}$ R. Berger et al., Microelectron. Eng. 35, 373 (1997).

${ }^{2}$ M. Papi et al., Appl. Phys. Lett. 88, 194102 (2006).

${ }^{3}$ D. Rugar et al., Nature (London) 430, 329 (2004).

${ }^{4}$ F. Kienberger et al., Acc. Chem. Res. 39, 29 (2006).

${ }^{5}$ B. Ilic et al., Nano Lett. 5, 925 (2005).

${ }^{6} \mathrm{M}$. Li et al., Nat. Nanotechnol. 2, 114 (2006).

${ }^{7}$ P. K. Hansma et al., Appl. Phys. Lett. 64, 1738 (1994).

${ }^{8}$ J. Tamayo, J. Struct. Biol. 141, 198 (2003).

${ }^{9}$ T. Braun et al., Phys. Rev. E 72, 031907 (2005).

${ }^{10}$ D. Ramos et al., J. Appl. Phys. 100, 106105 (2006).

${ }^{11}$ J. Tamayo et al., Appl. Phys. Lett. 89, 224104 (2006).

${ }^{12}$ J. Tamayo et al., Appl. Phys. Lett. 77, 582 (2000).

${ }^{13}$ W. Pang et al., Appl. Phys. Lett. 88, 243503 (2006).

${ }^{14}$ T. P. Burg et al., Nature (London) 446, 1066 (2007).

${ }^{15}$ D. Ebeling et al., Nanotechnology 17, S221 (2006).
}

${ }^{16}$ T. Fukuma et al., Appl. Phys. Lett. 86, 193108 (2005).

${ }^{17}$ M. J. Higgins et al., Biophys. J. 90, 640 (2006).

${ }^{18}$ A. Mehta et al., Appl. Phys. Lett. 78, 1637 (2001).

${ }^{19}$ J. Bechhoefer, Rev. Mod. Phys. 77, 783 (2005).

${ }^{20}$ O. Arcizet et al., Nature (London) 444, 71 (2006).

${ }^{21}$ J. Tamayo et al., Ultramicroscopy 86, 167 (2001).

${ }^{22}$ B. D’Urso et al., Phys. Rev. Lett. 94, 113002 (2005).

${ }^{23}$ M. Lax, Phys. Rev. 160, 290 (1967).

${ }^{24}$ D. Ham and A. Hajimiri, IEEE J. Solid-State Circuits 38, 407 (2003).

${ }^{25}$ J. Tamayo, J. Appl. Phys. 97, 044903 (2005).

${ }^{26}$ U. Dürig et al., J. Appl. Phys. 72, 1778 (1992).

${ }^{27}$ M. R. Paul and M. C. Cross, Phys. Rev. Lett. 92, 235501 (2004).

${ }^{28}$ J. E. Sader, J. Appl. Phys. 84, 64 (1998).

${ }^{29}$ A. D. L. Humphries et al., Langmuir 16, 7891 (2000). 